\title{
Leukotriene $B_{4}$ receptor 2 gene polymorphism (rs1950504, Asp196Gly) leads to enhanced cell motility under low-dose ligand stimulation
}

\author{
Jae-Hyun Jang ${ }^{1,4}$, Jun-Dong Wei ${ }^{1,4}$, Minsup Kim ${ }^{2}$, Joo-Young Kim ${ }^{3}$, Art E Cho ${ }^{2}$ and Jae-Hong Kim ${ }^{1}$
}

Recently, single-nucleotide polymorphisms (SNPs) in G-protein-coupled receptors (GPCRs) have been suggested to contribute to physiopathology and therapeutic effects. Leukotriene $B_{4}$ receptor 2 (BLT2), a member of the GPCR family, plays a critical role in the pathogenesis of several inflammatory diseases, including cancer and asthma. However, no studies on BLT2 SNP effects have been reported to date. In this study, we demonstrate that the BLT2 SNP (rs1950504, Asp196Gly), a Gly-196 variant of BLT2 (BLT2 D196G), causes enhanced cell motility under low-dose stimulation of its ligands. In addition, we demonstrated that Akt activation and subsequent production of reactive oxygen species (ROS), both of which act downstream of BLT2, are also increased by BLT2 D196G in response to low-dose ligand stimulation. Furthermore, we observed that the ligand binding affinity of BLT2 D196G was enhanced compared with that of BLT2. Through homology modeling analysis, it was predicted that BLT2 D196G loses ionic interaction with R197, potentially resulting in increased agonist-receptor interaction. To the best of our knowledge, this report is the first to describe a SNP study on BLT2 and shows that BLT2 D196G enhances ligand sensitivity, thereby increasing cell motility in response to low-dose ligand stimulation.

Experimental \& Molecular Medicine (2017) 49, e402; doi:10.1038/emm.2017.192; published online 24 November 2017

\section{INTRODUCTION}

G-protein-coupled receptors (GPCRs) are the largest human transmembrane receptor family and regulate various biological functions in mammals. ${ }^{1}$ GPCRs are targets for $\sim 36 \%$ of recently marketed drugs for treating human diseases, including neurological disorders, inflammatory diseases and cancer; ${ }^{2-4}$ however, genetic variation within GPCRs leads to variable drug responses. ${ }^{5}$ Single-nucleotide polymorphisms (SNPs) are genetic variations that, at the amino-acid level, can lead to functional changes in proteins and alterations of their responses to ligands. ${ }^{6,7}$ Given that SNPs contribute to physiopathology and therapeutic effects, the study of SNPs in GPCRs is critical to the understanding of human physiology.

Leukotriene $\mathrm{B}_{4}\left(\mathrm{LTB}_{4}\right)$ is a potent pro-inflammatory lipid mediator that is biosynthesized from arachidonic acid. ${ }^{8} \mathrm{LTB}_{4}$ exerts its biological functions via two types of GPCRs: a highaffinity $\mathrm{LTB}_{4}$ receptor (BLT1) and a low-affinity $\mathrm{LTB}_{4}$ receptor (BLT2). ${ }^{9}$ Unlike BLT1, BLT2 is minimally expressed under normal homeostatic conditions but is specifically overexpressed under stress-induced inflammatory conditions; ${ }^{10-13}$ however, the physiological function of BLT2 is largely unknown. Recent reports have demonstrated that the activation of BLT2 through ligand stimulation is associated with chemotaxis in primary keratinocytes. ${ }^{14}$ Previously, we have observed that the $\mathrm{LTB}_{4}{ }^{-}$ BLT2 axis is associated with the generation of reactive oxygen species (ROS). ${ }^{15}$ Additionally, the overexpression of BLT2 causes an $\mathrm{LTB}_{4}$-induced increase in chemotactic responses in a ROS-dependent manner. ${ }^{16,17}$ In addition, activation of Akt by $\mathrm{LTB}_{4}$, which phosphorylates BLT2 at $\mathrm{Thr}^{355}$, is critical for BLT2-mediated chemotactic responses. ${ }^{17}$ Furthermore, recent studies have indicated that the $\mathrm{LTB}_{4}$-BLT2 axis is critical for the pathogenesis of inflammatory diseases, including asthma, ${ }^{13,18}$ carotid atherosclerosis, ${ }^{19,20}$ and cancer. ${ }^{12,21-25}$ Despite the critical suggested role of BLT2 in various human diseases, there have been no reports on SNP effects in BLT2.

In this study, we observed that BLT2 D196G enhanced BLT2-mediated cell motility under low-dose ligand stimulation. We demonstrated that BLT2 D196G also increased the generation of ROS and activation of AKT (p-AKT), thus enhancing cell motility. In addition, molecular modeling

\footnotetext{
${ }^{1}$ Department of Biotechnology, College of Life Sciences, Korea University, Seoul, Korea; ${ }^{2}$ Department of Bioinformatics, Korea University, Sejong, Korea and ${ }^{3}$ Department of Crime-Scene DNA Section, Gwangju Institute, National Forensic Service, Gwangju, Korea

${ }^{4}$ These authors contributed equally to this work.

Correspondence: Professor J-H Kim, College of Life Sciences and Biotechnology, Korea University, 5-1 Anam-dong, Sungbuk-gu, Seoul 02841, Korea.

E-mail: jhongkim@korea.ac.kr

Received 27 December 2016; revised 17 May 2017; accepted 29 May 2017
} 
analysis predicted that BLT2 D196G undergoes a conformational change affecting the side chain Arg197, which is important for the ionic interaction with BLT2 ligands, thereby increasing ligand binding affinity. Taken together, our results constitute the first SNP study on BLT2 and show that BLT2 D196G enhances ligand sensitivity and binding affinity, thereby contributing to increased BLT2-mediated cell motility.

\section{MATERIALS AND METHODS}

\section{Chemicals and plasmids}

RPMI 1640 medium was purchased from Corning (Corning Inc., Corning, NY, USA). Phenol red-free RPMI 1640 medium was purchased from Welgene (Gyeongsan, Korea). Fetal bovine serum (FBS) was purchased from HyClone (Logan, UT). $\mathrm{LTB}_{4}, 12(\mathrm{~S})$ hydroxyheptadeca-5Z, 8E, 10E-trienoic acid (12-HHT), and 12(S)hydroxy-5Z, 8Z, 10E, 14Z-eicosatetraenoic acid (12(S)-HETE) were purchased from Cayman (Ann Arbor, MI). The human BLT2 (GenBank accession no. NM_019839.1) plasmid was cloned through PCR using a human genomic bacterial artificial chromosome (BAC) library with minor modifications to the PCR conditions. ${ }^{26}$ The BAC library was a kind gift from Dr Young-Chul Choi (Kyung Hee University, Korea). The pcDNA3.1 vectors were purchased from Invitrogen (Carlsbad, CA, USA). 2',7'-Dichlorodihydro-fluorescein diacetate $\left(\mathrm{H}_{2} \mathrm{DCFDA}\right)$ was purchased from Molecular Probes (Eugene, OR, USA). Anti-Akt antibody was obtained from Cell Signaling Technology (Danvers, MA, USA). All other chemicals were obtained from standard sources and were of molecular biology grade or higher.

\section{Site-directed mutagenesis}

The constructs with point mutations in BLT2 D196G were obtained by site-directed mutagenesis PCR using the following primers: forward for D196G (5'-CTGTGGAGGGGCCGCGTA-3') and reverse for D196G (5'-ATACGCGGCCCCTCCACA-3'). The first PCR products for site-directed mutagenesis were obtained using the forward primer for BLT2 (5'-CTGGATCCCACCATGTACCCCTACGACGTGCCCG ACTACGCCGCACCTTCTCATCGG-3') and the reverse D196G primers. The second PCR products were obtained using the forward D196G primers and reverse primer for BLT2 (5'-GGTGAATTCT CAAA GGTCCCATTCCGG-3'). The final BLT2 D196G products were constructed using PCR amplification with forward and reverse primers for BLT2. The BLT2 D196G product was cloned into pcDNA3.1 and was confirmed by DNA sequencing.

\section{Cell culture and DNA transfection}

CHO-K1 cells were obtained from the Korean Cell Line Bank (KCLB, 10061), and the cells were grown in RPMI 1640 medium supplemented with 10\% FBS and antibiotic-antimycotic solution (Gibco, El Paso, TX) at $37^{\circ} \mathrm{C}$ in a $5 \% \mathrm{CO}_{2}$ humidified atmosphere. Transient transfection was performed by plating $2 \times 10^{5}$ cells in 60 -mm dishes for $24 \mathrm{~h}$ and then adding Lipofectamine (4 $\mu \mathrm{l}$; Invitrogen) and DNA $(2 \mu \mathrm{g})$ to each dish. The total transfected DNA quantities were equalized in each experiment based on the pcDNA3.1 vector DNA.

\section{Semi-quantitative reverse transcription-PCR}

Total RNA was extracted from cells using EasyBlue (Intron, Sungnam, Korea), and a portion $(1.25 \mu \mathrm{g})$ of the RNA was subjected to reverse transcription (RT) with M-MLV reverse transcriptase (Beams Bio, Gyunggi, Korea) followed by semi quantitative PCR analysis with a PCR PreMix Kit (Intron) under optimal conditions for linear amplification of GAPDH cDNA. The primer sequences used were as follows: human BLT2 (forward, 5'-AGCCTGGAGACTCTGACC GCTTTCG-3'; reverse, 5'-GACGTAGCACCGGGTTGACGCTA-3') and GAPDH (forward, 5'-CTGCACCACCAACTGCTTAGC-3'; reverse, $5^{\prime}$-CTTCACCACCTTCTTGATGTC- $3^{\prime}$ ). The specificity of all primers was confirmed by sequencing the PCR products. The final PCR products were resolved on a $1.5 \%$ agarose gel containing ethidium bromide and were visualized under UV light.

\section{Flow cytometry analysis}

The expression of HA-tagged BLT2 or BLT2 D196G constructs in CHO-K1 cells was confirmed by staining the cells with antibody against the $\mathrm{HA}$ epitope added to the $\mathrm{NH}_{2}$-terminus of the receptors. The cells were fixed with phosphate-buffered saline (PBS) containing $2 \%(\mathrm{w} / \mathrm{v})$ paraformaldehyde, and then were incubated on a rotating wheel for $40 \mathrm{~min}$ at $4{ }^{\circ} \mathrm{C}$, followed by blocking with PBS containing $2 \%$ BSA (Fraction V, Sigma-Aldrich, St Louis, MO, USA). Cells were then incubated with $4 \mu \mathrm{g} \mathrm{ml}^{-1} \mathrm{HA}$-specific antibody or $4 \mu \mathrm{g} \mathrm{ml}^{-1}$ normal mouse IgG (Sigma-Aldrich) in PBS containing 2\% BSA for $40 \mathrm{~min}$ at room temperature followed by staining with fluorescein isothiocyanate-conjugated anti-mouse IgG (1:200 dilutions) for $30 \mathrm{~min}$ at room temperature in the dark. The cells were washed twice with PBS and were analyzed with a FACSCalibur flow cytometer (Becton Dickinson, Franklin Lakes, NJ, USA).

\section{Chemotaxis assay}

Cells were assayed using Transwell chambers with 6.5 -mm-diameter polycarbonate filters (8- $\mu \mathrm{m}$ pore size). Briefly, the lower surfaces of the filters were coated with $1 \mathrm{mg} \mathrm{ml}^{-1}$ fibronectin (Roche Applied Science, Indianapolis, IN, USA) in serum-free RPMI 1640 medium for $2 \mathrm{~h}$ at $37^{\circ} \mathrm{C}$. Dry-coated filters containing various amounts of $\mathrm{LTB}_{4}$, 12-HHT, or 12(S)-HETE were placed in the lower wells of the Transwell chambers. Next, $100 \mu \mathrm{l}$ of CHO-BLT2 or CHO-D196G cells in serum-free RPMI was loaded into the top wells, thus yielding a final concentration of $1 \times 10^{6}$ cells per ml. After incubation at $37^{\circ} \mathrm{C}$ in $5 \%$ $\mathrm{CO}_{2}$ for $3 \mathrm{~h}$, the filters were disassembled, and the upper surface of each filter was scraped free of cells through wiping with a cotton swab. Cells that migrated to the underside of the filter were fixed for $5 \mathrm{~min}$ with methanol and were stained for 3 min with eosin and hematoxylin. Chemotaxis was quantified by counting the cells on the lower side of the filter under an optical microscope (magnification, $\times 200$ ). Five fields were counted in each assay. Each sample was assayed in duplicate, and the assays were repeated three times.

\section{Cell migration assay}

Cells were seeded onto a six-well culture plate and were incubated in a standard $\mathrm{CO}_{2}$ incubator until $80 \%$ confluency was reached, and then wounds were generated with a 200p pipette tip. The wounded cells were washed twice and were subsequently treated with each concentration of ligand containing RPMI medium with 1\% FBS. Photographs of the cultures were obtained immediately after scratching and $24 \mathrm{~h}$ later in the same position. Four fields were analyzed in each assay. Each sample was assayed in duplicate, and the assays were repeated three times.

\section{Western blotting}

Cells were collected and lysed with buffer (40 mM Trish-HCl, pH 8.0, $120 \mathrm{~mm} \mathrm{NaCl}, \quad 0.1 \%$ Nonidet-P40, $100 \mathrm{~mm}$ phenylmethylsulfonyl fluoride, $1 \mathrm{~mm}$ sodium orthovanadate, $2 \mathrm{~g} \mathrm{ml}^{-1}$ leupeptin, and $2 \mathrm{~g} \mathrm{ml}^{-1}$ aprotinin, phosSTOP cocktail). Proteins were separated by 
SDS-polyacrylamide gel electrophoresis and were transferred to a polyvinylidene fluoride membrane. The membrane was blocked with $5 \%$ nonfat dry milk in Tris-buffered saline and was incubated with primary antibodies against $\mathrm{p}-\mathrm{AKT}$ or AKT for $1 \mathrm{~h}$ at room temperature. Blots were developed with a peroxidase-conjugated secondary antibody, and proteins were visualized by enhanced chemiluminescence (Amersham Biosciences, UK).

\section{Measurement of ROS}

Cells $\left(3 \times 10^{5}\right.$ per plate $)$ were plated in $60-\mathrm{mm}$ dishes and incubated in RPMI supplemented with $10 \%$ FBS for $24 \mathrm{~h}$ before measurement of ROS. To measure intracellular $\mathrm{H}_{2} \mathrm{O}_{2}$, actively growing cells were incubated for $20 \mathrm{~min}$ in the dark at $37^{\circ} \mathrm{C}$ with $20 \mu \mathrm{M}$ DCF-DA. DCFDA is hydrolyzed intracellularly to DCF, which is trapped inside the cell. Although DCF-DA itself is not fluorescent, it is oxidized in the presence of $\mathrm{H}_{2} \mathrm{O}_{2}$ to the highly fluorescent DCF. After being loaded with the indicator, the cells were collected using trypsin-EDTA and were resuspended in phenol-red free RPMI. After incubation, cells were treated with each concentration of ligand for $15 \mathrm{~min}$, and DCF fluorescence was measured using a FACSCalibur flow cytometer. The values represent the means \pm s.d. of DCF fluorescence from three independent experiments.

\section{Ligand-binding assay}

Cells were grown to near confluence and were collected with PBS. Cells were resuspended in buffer A (50 mm Tris-Cl, pH 7.7, 5 mм EDTA) and homogenized in a glass homogenizer (30 s, repeated 5 times). The homogenates were first centrifuged at $1000 \mathrm{~g}$ for $5 \mathrm{~min}$ at $4{ }^{\circ} \mathrm{C}$, and the resulting supernatants were re-centrifuged at $45000 \mathrm{~g}$ for

Table 1 BLT2 gene variations in order of minor allele frequency

\begin{tabular}{|c|c|c|c|c|}
\hline Variant ID & $\begin{array}{c}\text { Global MAF } \\
\text { (\%) }\end{array}$ & $\begin{array}{c}\text { Alleles } \\
\text { Major/minor }\end{array}$ & $\begin{array}{c}A A \\
\text { Co-ordinate }\end{array}$ & Resulting $A A$ \\
\hline rs1950504 & 3.8 & $A / G$ & 196 & $D / G$ \\
\hline rs1950503 & 2.5 & $\mathrm{C} / \mathrm{A} / \mathrm{T}$ & 219 & $\mathrm{~T}$ \\
\hline rs150416614 & 1.6 & $\mathrm{G} / \mathrm{A}$ & 80 & $\mathrm{R} / \mathrm{Q}$ \\
\hline rs78449402 & 0.8 & $\mathrm{G} / \mathrm{C}$ & 381 & $E / Q$ \\
\hline rs138224360 & 0.7 & $\mathrm{C} / \mathrm{T}$ & 161 & $\mathrm{P} / \mathrm{S}$ \\
\hline rs115630307 & 0.5 & $\mathrm{C} / \mathrm{A} / \mathrm{T}$ & 52 & $A$ \\
\hline rs151291081 & 0.5 & $\mathrm{G} / \mathrm{A}$ & 10 & V/M \\
\hline rs77369205 & 0.4 & $\mathrm{C} / \mathrm{T} / \mathrm{A}$ & 77 & AN/E \\
\hline rs199715515 & 0.3 & $\mathrm{G} / \mathrm{A}$ & 251 & $\mathrm{~A} / \mathrm{T}$ \\
\hline rs148153989 & 0.3 & $\mathrm{~A} / \mathrm{T}$ & 380 & $M / L$ \\
\hline rs375081944 & 0.2 & $\mathrm{G} / \mathrm{A}$ & 78 & $\mathrm{G} / \mathrm{S}$ \\
\hline rs201778907 & 0.2 & $A / G$ & 147 & $S / G$ \\
\hline rs146912351 & 0.2 & $T / G$ & 3 & $\mathrm{P}$ \\
\hline rs199950899 & 0.2 & $A / G$ & 325 & $D / G$ \\
\hline rs377045409 & 0.2 & $\mathrm{C} / \mathrm{G} / \mathrm{T}$ & 358 & $\mathrm{~L} / \mathrm{N} / \mathrm{F}$ \\
\hline rs370582090 & 0.1 & $\mathrm{C} / \mathrm{T}$ & 179 & A \\
\hline rs145778310 & 0.1 & $\mathrm{~A} / \mathrm{C}$ & 324 & $G$ \\
\hline rs562644365 & 0.1 & $\mathrm{G} / \mathrm{A} / \mathrm{C}$ & 252 & $\mathrm{R} / \mathrm{Q} / \mathrm{P}$ \\
\hline rs143666989 & 0.1 & $\mathrm{~A} / \mathrm{G}$ & 363 & $\mathrm{Q} / \mathrm{R}$ \\
\hline
\end{tabular}

Abbreviations: AA, amino acid; Alleles, alternative nucleotides; Global MAF, Global minor allele frequency.

The human BLT2 SNP data were obtained from Ensembl (http://www.ensembl.org/). In Ensembl, the Global MAF is calculated using the allele frequencies across all 1000 Genomes Phase 3 populations.
40 min at $4{ }^{\circ} \mathrm{C}$. The cell membrane pellet was washed in buffer $\mathrm{A}$, and each sample was adjusted to a protein concentration of $40 \mathrm{mg} \mathrm{ml}^{-1}$. The samples were incubated with $5 \mathrm{~nm}\left[{ }^{3} \mathrm{H}\right]-(+)$-leukotriene $\mathrm{B}_{4}$ radioligand $(120 \mathrm{Ci} / \mathrm{mmol}$; American Radiolabeled Chemicals, Saint Louis, MO, USA ) and ligands $(0.1 \mathrm{~nm}-1 \mu \mathrm{M})$ for $60 \mathrm{~min}$ at $37^{\circ} \mathrm{C}$. The reaction was stopped by rapid filtration through a Whatman $\mathrm{GF} / \mathrm{B}$ glass fiber filter followed by washing with cold buffer ( $50 \mathrm{~mm}$ Tris-Cl, $\mathrm{pH}$ 7.7) using a Brandel cell harvester. The filters were then immersed in scintillation cocktail, and the radioactivity of each filter was determined using a Hidex 300sl liquid scintillation counter. Binding parameters were determined with Prism software (GraphPad Software, Inc., San Diego, CA, USA) using nonlinear regression.

\section{Molecular modeling}

The three-dimensional structures of BLT2 !and BLT2 D196G were predicted using the Prime homology modeling tool (Prime, Schrödinger, LLC, New York, NY, USA, 2016), and binding poses of $\mathrm{LTB}_{4}$ were predicted using the protein-ligand docking software SolvQMDock. ${ }^{27}$ Prime MM-GB/SA (Molecular Mechanics-Generalized Born/Surface Area) was used to calculate the binding free energy of bound ligand.

\section{Data analysis and statistics}

All values are expressed as the means \pm s.d. Statistical comparisons between experimental groups were performed using Student's $t$-test. Values of $P<0.01$ were considered to be statistically significant. Values of $P<0.01, P<0.005$, and $P<0.001$ are designated by ${ }^{*},{ }^{* *}$, and ${ }^{* *}$, respectively.

\section{RESULTS}

SNP variants within the human BLT2 coding region

To study the polymorphisms of the BLT2 gene, we first searched for SNPs within BLT2 using Ensembl. According to the Ensembl 84 release $^{28}$ from March 2016, there are 19 variants with minor allele frequencies (MAFs) $>0.1 \%$ in human BLT2 (Table 1). Moreover, there are three SNPs occurring in a coding region with a MAF greater than $1 \%$ : rs1950504, rs1950503 and rs150416614. Among these SNPs, rs1950504 exhibited the greatest MAF (0.039) within the BLT2 coding region and is characterized by a single base-pair transition $(A \rightarrow G)$ at nucleotide 196 , thus resulting in a nonsynonymous amino-acid substitution of Asp196Gly (designated as D196G; Table 1). To study the function of BLT2 SNP (D196G), we first generated BLT2 D196G by site-directed mutagenesis (Figure 1a), and we assessed whether BLT2 D196G shows any altered expression levels in CHO-K1 cells. Both semi-quantitative reverse transcription PCR (Figure 1b) and FACS analysis (Figure 1c) revealed that the expression levels of BLT2 were similar in cells transfected with BLT2 or BLT2 D196G, suggesting that this SNP does not affect BLT2 expression (Figure 1).

\section{BLT2 D196G leads to enhanced migration in response to low-dose ligands}

Recent studies have demonstrated that BLT2 regulates cell motility, including migration and chemotaxis. ${ }^{16}$ Therefore, we first examined whether BLT2 D196G could cause any change in chemotactic migration in response to its ligand $\mathrm{LTB}_{4}$. To this 


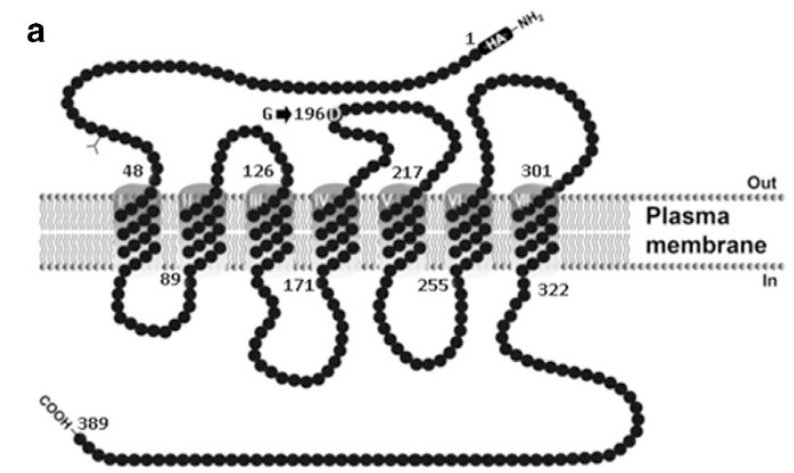

b

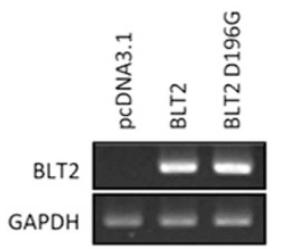

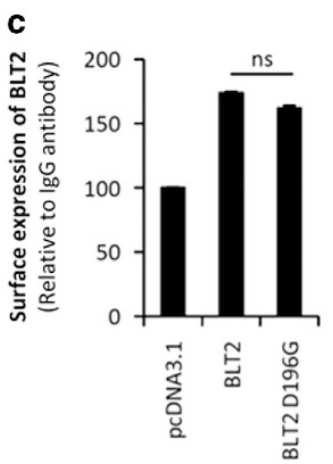

Figure 1 Snake plot of BLT2 D196G and its expression in CHO-K1 cells. (a) Topology model of BLT2 D196 in which the second extracellular loop was replaced by Gly-196. The amino-acid residues were exchanged for glycine by site-directed mutagenesis. (b) CHO-K1 cells were transiently transfected with BLT2, BLT2 D196G or control vector. Twenty-four hours after transfection, total RNA was isolated and subjected to semiquantitative reverse transcription PCR (RT-PCR) analysis of BLT2 mRNAs. Data are representative of three independent experiments with similar results. (c) CHO-K1 cells were transiently transfected with BLT2, BLT2 D196G or control vector. Twenty-four hours after transfection, cells were stained with primary anti-HA antibodies against human BLT2 and were analyzed by flow cytometry on a FACS Calibur instrument. The data are represented as means \pm s.d.; $n=3$ independent experiments. ns: not significant according to Student's $t$-test.

end, we performed chemotactic migration analysis using the Transwell assay in CHO-K1 cells transiently transfected with the expression plasmid for BLT2 or BLT2 D196G. Previously, the concentration of the BLT2 ligands used for chemotaxis experiments in CHO-BLT2 cells was $100-300 \mathrm{~nm}$, and low doses, such as $10 \mathrm{~nm}$, showed no significant effects in these BLT2-expressing cells. ${ }^{16,26,29}$ Thus, we defined the $10 \mathrm{~nm}$ concentration as the low-dose ligand condition. As shown in Figure 2a, the BLT2 ligand $\mathrm{LTB}_{4}$-enhanced chemotaxis in cells expressing BLT2 D196G compared with cells expressing wildtype BLT2 under low-dose conditions. Similar enhanced chemotactic effects were observed for BLT2 D196G in response to other types of BLT2 ligands, including 12-HHT or 12(S)HETE, under low-dose conditions (Figures $2 b$ and $c$ ). To further analyze the enhanced motility mediated by BLT2 D196G, we examined whether BLT2 D196G enhanced wound migration under low-dose ligand concentrations. Clearly, cells transfected with BLT2 D196G elicited significantly increased motility under low-dose ligand conditions (Figure 3). Thus, our results suggest that BLT2 D196G enhances cell motility under low-dose ligand conditions.

\section{BLT2 D196G leads to enhanced ROS generation and AKT} phosphorylation under low-dose ligand conditions

Recent studies have demonstrated that the activation of BLT2 induces the generation of intracellular ROS which, in turn, plays a critical role in cell motility. ${ }^{15,16,26}$ Therefore, we determined whether BLT2 D196G leads to enhanced ROS generation. Enhanced $\mathrm{LTB}_{4}$-elicited ROS generation was clearly observed in cells transfected with BLT2 D196G (Figures $4 \mathrm{a}$ and $\mathrm{b}$ ) under low-dose ligand conditions. Similarly, we observed enhanced effects in BLT2 D196G in response to 12-HHT or 12(S)-HETE stimulation (Figures $4 \mathrm{c}$ and d). We have previously reported that BLT2-mediated ROS generation and chemotactic motility are regulated by AKT phosphorylation. ${ }^{16,17,26}$ Therefore, we next assessed whether BLT2 D196G leads to enhanced AKT phosphorylation. As shown in Figure 5a, AKT phosphorylation was significantly enhanced (approximately twofold increase) in cells expressing BLT2 D196G and treated with low-dose $\mathrm{LTB}_{4}$ or 12-HHT stimulation (Figure 5b) compared with BLT2-transfected cells. Together, these results suggest that BLT2 D196G causes enhanced ROS generation and Akt activation, thereby increasing cell migration under low-dose ligand conditions.

BLT2 D196G leads to enhanced ligand binding affinity under low-dose ligand conditions

To understand the mechanism by which BLT2 D196G exhibits hypersensitivity to low doses of its ligands, we tested whether BLT2 D196G exhibits higher ligand-binding affinity than BLT2, especially under low-dose ligand conditions. By measuring the ability of BLT2 ligands to compete for ${ }^{3} \mathrm{H}-\mathrm{LTB}_{4}$ binding to the membranes of cells expressing BLT2 or BLT2 D196G, we clearly observed that membranes with BLT2 D196G exhibited significantly enhanced binding affinity to $\mathrm{LTB}_{4}$ at low doses (Figure 6a). Similarly, membranes with BLT2 D196G exhibited similar enhanced binding affinity to 12 -HHT and $12(S)$-HETE at low doses (Figures $6 \mathrm{~b}$ and c). Taken together, these data indicate that BLT2 D196G leads to enhanced ligand binding affinities, especially under low-dose ligand conditions.

Structural molecular modeling study of BLT2 with its ligand To understand the molecular mechanism of how BLT2 D196G exhibits enhanced ligand binding affinity under low-dose conditions, we performed molecular modeling analysis for this SNP. Using homology modeling methods, we compared the three-dimensional structures of BLT2 and BLT2 D196G and predicted the binding poses of $\mathrm{LTB}_{4}$ for each structure using docking simulations. In the predicted binding pose, $\mathrm{LTB}_{4}$ forms ionic bonds with $\mathrm{H} 203$ and R197 located within extracellular loop 2 (ECL2) in BLT2 or BLT2 D196G (Figures 7a and b). R197 in BLT2 forms another ionic bond with D196, thus limiting the favorable binding to $\mathrm{LTB}_{4}$; 
a

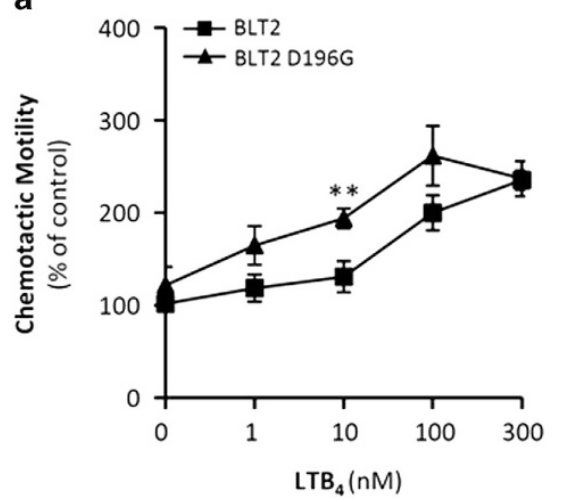

b

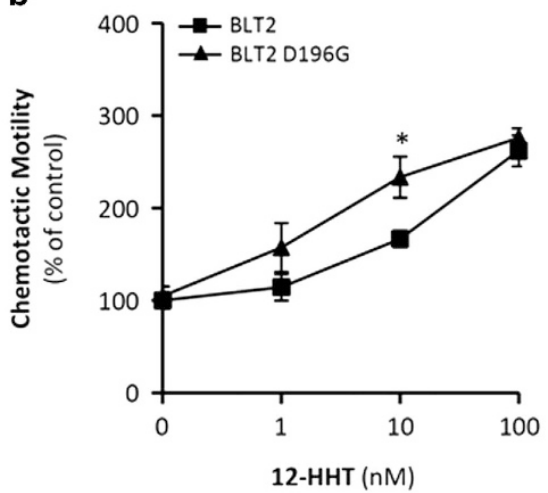

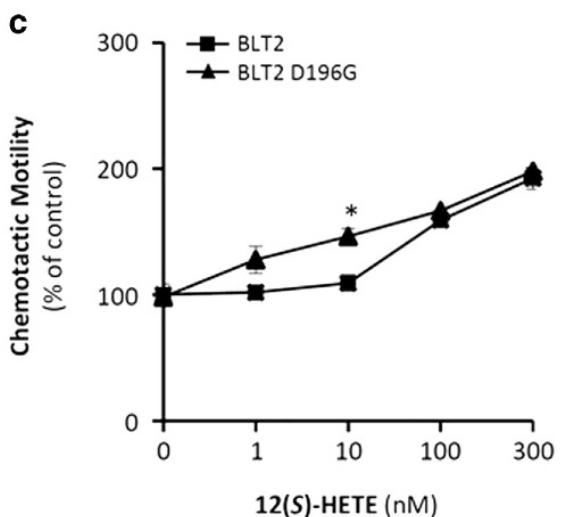

Figure 2 BLT2 D196G leads to enhanced chemotactic motility under low-dose ligand stimulation. (a-c) CHO-K1 cells were transiently transfected with BLT2 or BLT2 D196G. Forty-eight hours after transfection, the transfected cells were exposed to each concentration of $\mathrm{LTB}_{4}(\mathbf{a}), 12-\mathrm{HHT}$ (b) or 12(S)-HETE (c) for $2 \mathrm{~h}$. The migrating cells were then fixed and stained with hematoxylin and eosin. Migratory activity is expressed as a fold-change relative to $0 \mathrm{~nm}$ of each ligand, and the data are represented as means \pm s.d.; $n=3$ independent experiments. ${ }^{*} P<0.01$ and ${ }^{* *} P<0.005$ according to Student's $t$-test. The $t$-test was performed to determine whether the difference was significant between BLT2 and BLT2 D196G with a ligand concentration of $10 \mathrm{~nm}$.

a

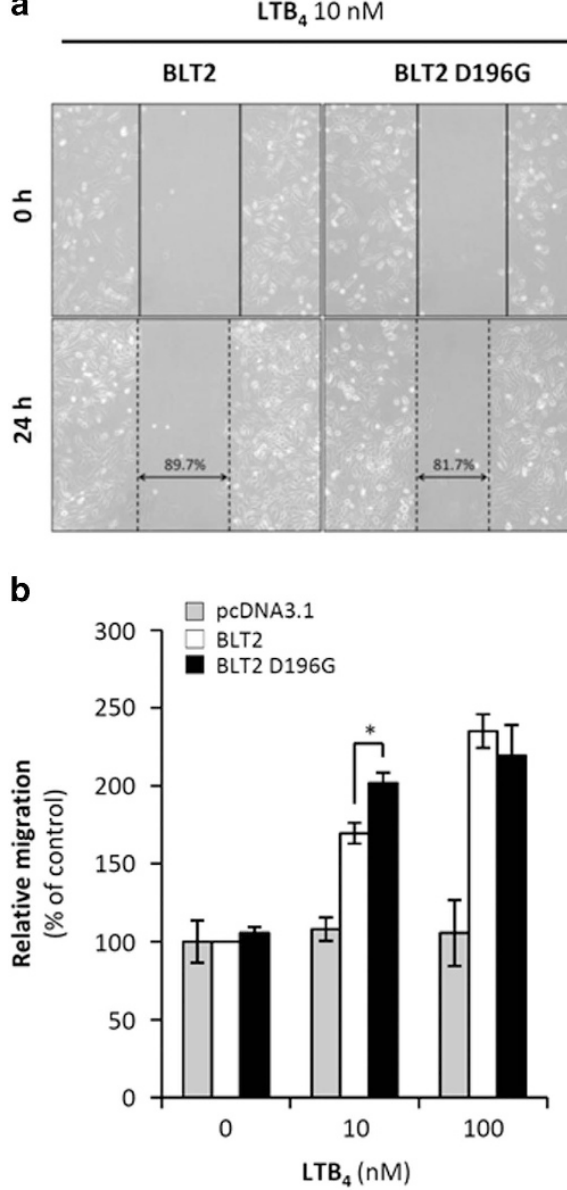

12-HHT $10 \mathrm{nM}$

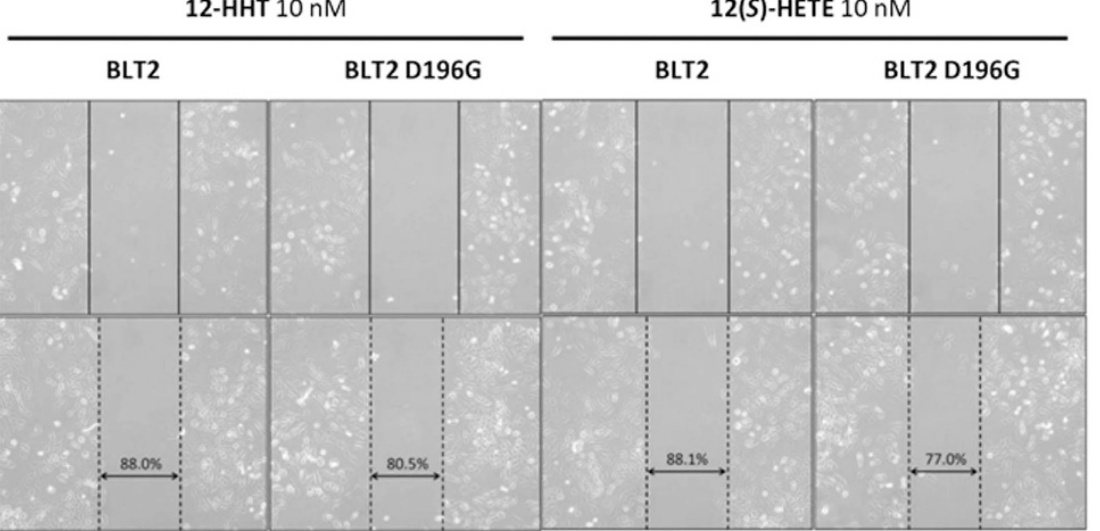

C

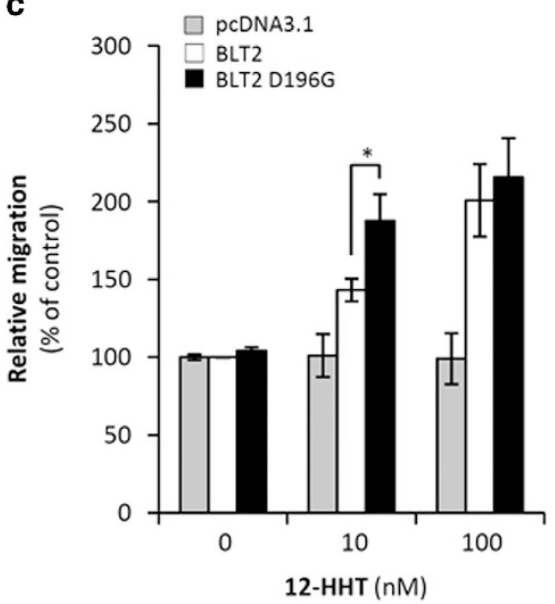

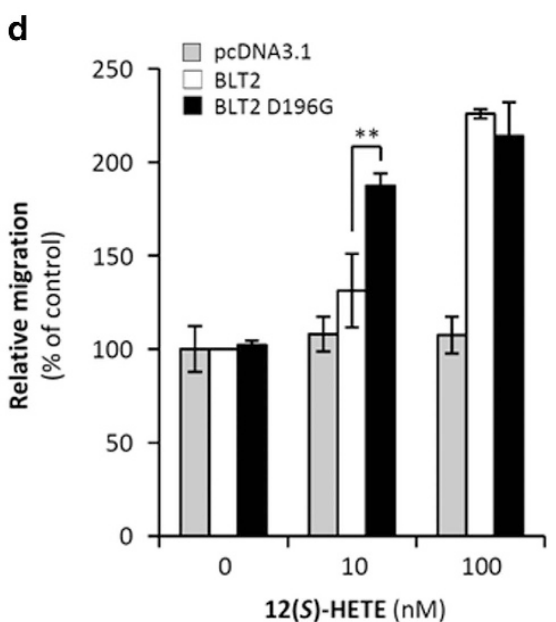

Figure 3 BLT2 D196G leads to enhanced migration under low-dose ligand stimulation. (a-d) CHO-K1 cells were seeded onto 6-well culture plates and then were transiently transfected with BLT2 or BLT2 D196G. Cells were then incubated in a standard $\mathrm{CO}_{2}$ incubator to $80 \%$ confluency, and then wounds were generated with a $200 p$ pipette tip. (a) The wounded cells were washed twice and then were treated with $10 \mathrm{~nm}$ of the indicated ligand-containing RPMI medium with $1 \%$ FBS. Photographs of the cultures were obtained immediately after scratching and at $24 \mathrm{~h}$ later in the same position. (b-d) The dose dependence of migration was determined after treatment with the indicated ligands, as described above. The results are expressed as a fold-change relative to $0 \mathrm{~nm}$ of each ligand, and the data are represented as means \pm s.d.; $n=3$ independent experiments. ${ }^{*} P<0.01$ and ${ }^{* *} P<0.005$ according to Student's $t$-test. The $t$-test was performed to determine whether the difference was significant between BLT2 and BLT2 D196G with a ligand concentration of $10 \mathrm{~nm}$. 


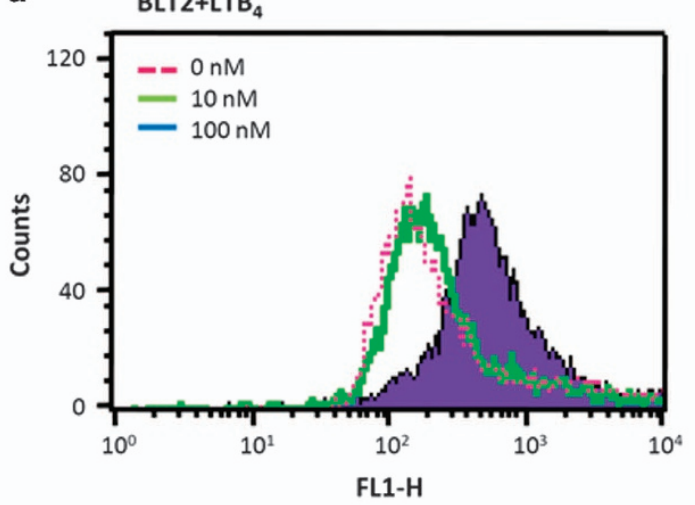

BLT2 D196G+LTB 4

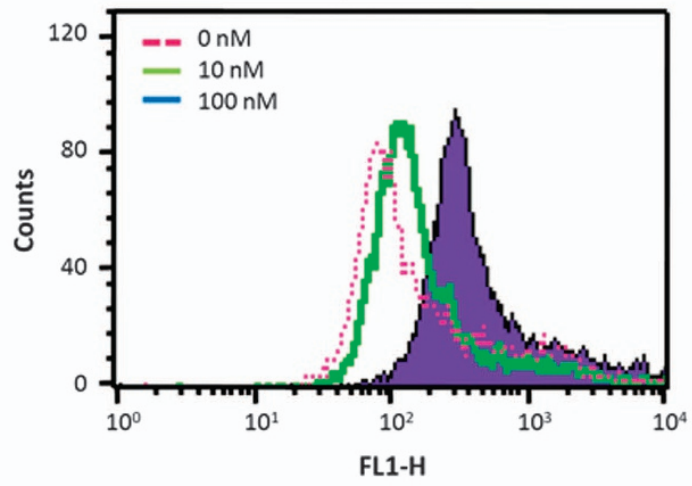

b

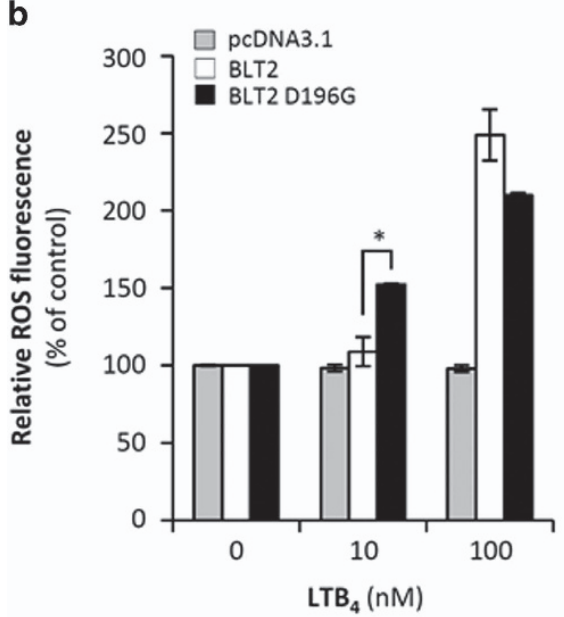

c

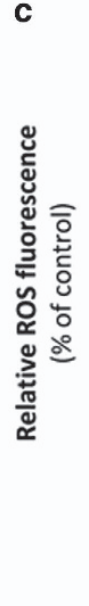

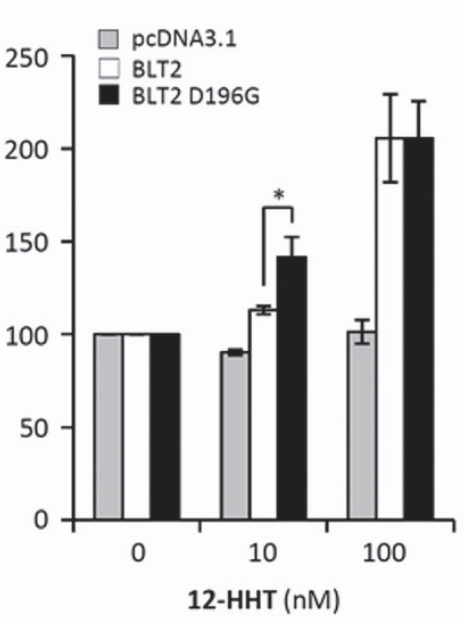

d

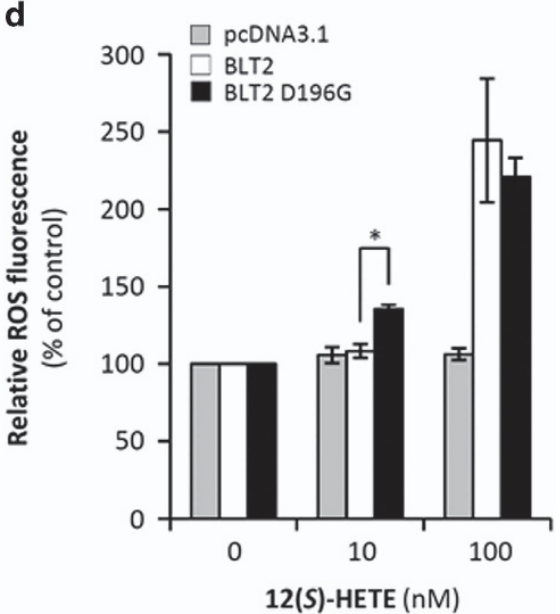

Figure 4 BLT2 D196G leads to enhanced BLT2-mediated ROS generation under low-dose ligand stimulation. (a-d) CHO-K1 cells were transiently transfected with BLT2 or BLT2 D196G. (a) Twenty-four hours after transfection, the transfected cells were stabilized in serumreduced phenol red-free RPMI 1640 medium for at least $3 \mathrm{~h}$ before exposure to the indicated concentration of $\mathrm{LTB}_{4}$. Cells were treated with $\mathrm{LTB}_{4}$ for $15 \mathrm{~min}$. Next, DCF fluorescence was monitored by flow cytometry as described in the Materials and Methods section. (b-d) Cells were treated with the indicated ligands for $15 \mathrm{~min}$ and then were assayed for ROS generation as described above. DCF fluorescence is expressed as the fold change relative to $0 \mathrm{~nm}$ of each ligand, and the data are presented as means \pm s.d.; $n=3$ independent experiments. ${ }^{*} P<0.01$ according to Student's $t$-test. The $t$-test was performed to determine whether the difference was significant between BLT2 and BLT2 D196G with a ligand concentration of $10 \mathrm{~nm}$.

however, R197 in BLT2 D196G does not maintain the ionic bond with G196, thereby affording more energetically favorable and stronger ionic bonding with its ligand $\mathrm{LTB}_{4}$. Indeed, our modeling analysis demonstrated that $\mathrm{LTB}_{4}$ exhibited more favorable binding free energy with BLT2 D196G, and the difference in predicted binding free energy $(\Delta \Delta G)$ was $-3.61 \mathrm{kcal} \mathrm{mol}^{-1}$.

\section{DISCUSSION}

In the present study, we observed that BLT2 D196G leads to enhanced chemotactic motility under low-dose ligand conditions. We also demonstrated that BLT2 D196G increased the generation of ROS and activation of AKT (p-AKT), thereby enhancing cell motility under low-dose ligand stimulation. In addition, we demonstrated that ligand binding affinity was enhanced in BLT2 D196G-transfected cells using a ${ }^{3} \mathrm{H}-\mathrm{LTB}_{4}$ radioligand binding assay. Finally, we found that D196G improves the conformational freedom of BLT2 Arg197, thus facilitating important ionic interactions with ligands.

BLT2 is a low-affinity GPCR for $\mathrm{LTB}_{4}$, which is a lipid metabolite of arachidonic acid derived via the 5-lipoxygenasedependent pathway. In most GPCRs, genetic variation within GPCRs leads to their cellular signaling transduction, phenotypes, and variable drug responses. ${ }^{5,30-32}$ To date, numerous non-synonymous genetic variations have been identified in humans. SNPs can be assigned a MAF, which is the less common allele frequency at a locus that is observed in a population. To study the genetic variation of BLT2, we first searched for SNP sites in BLT2 and found that the BLT2 SNP (rs1950504, D196G) exhibits a MAF of 0.039 (Table 1). Previously, we have demonstrated that BLT2 regulates cell motility. ${ }^{16,17}$ Thus, we examined whether BLT2 D196G affects BLT2-mediated cell motility. For these experiments, we prepared a mutant with glycine substituted for Asp-196. In agreement with the proposed role of BLT2 in cell motility, 
a
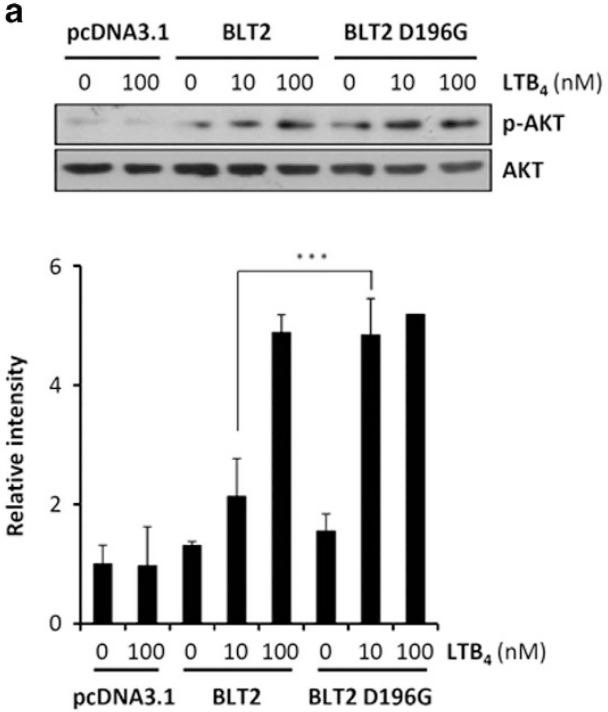

b

\begin{tabular}{|c|c|c|c|c|c|c|c|c|}
\hline \multicolumn{2}{|c|}{ pcDNA3.1 } & \multicolumn{3}{|c|}{ BLT2 } & \multicolumn{3}{|c|}{ BLT2 D196G } & \multirow[b]{2}{*}{ 12-HHT (nM) } \\
\hline 0 & 100 & 0 & 10 & 100 & 0 & & 100 & \\
\hline 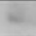 & $+\infty$ & & $\omega$ & ש & & 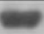 & 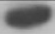 & p-AKT \\
\hline$=$ & - & & - & $=$ & $=$ & ${ }_{-}$ & & AKT \\
\hline
\end{tabular}

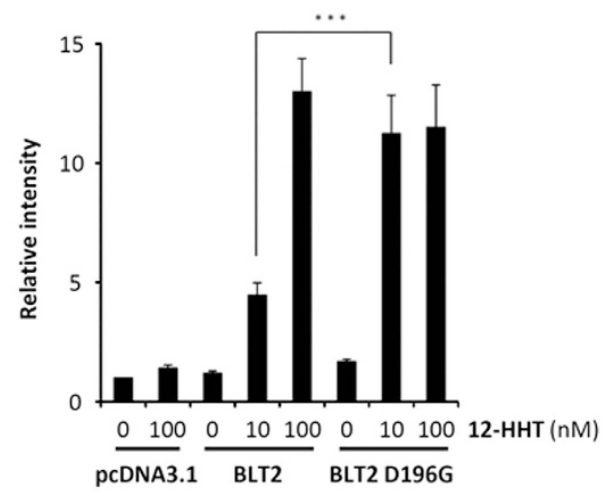

Figure 5 BLT2 D196G leads to enhanced AKT phosphorylation under low-dose ligand stimulation. (a, b) CHO-K1 cells were transiently transfected with BLT2, BLT2 D196G or control vector. (a) Twenty-four hours after transfection, the transfected cells were exposed to each concentration of $\mathrm{LTB}_{4}$ for $2 \mathrm{~min}$, and then the cell lysates were assayed for the level of Akt phosphorylation by immunoblotting as described in the Materials and Methods section. Data analysis was performed with the ImageJ program, and the data are presented as means \pm s.d.; $n=3$ independent experiments. ${ }^{* *} P<0.001$ according to Student's $t$-test. The $t$-test was performed to determine whether the difference was significant between BLT2 and BLT2 D196G with a ligand concentration of 10 nM. (b) Cells were treated with the indicated concentrations of $12-\mathrm{HHT}$ for $2 \mathrm{~min}$. Next, cell lysates were analyzed for Akt phosphorylation by immunoblotting as described above.
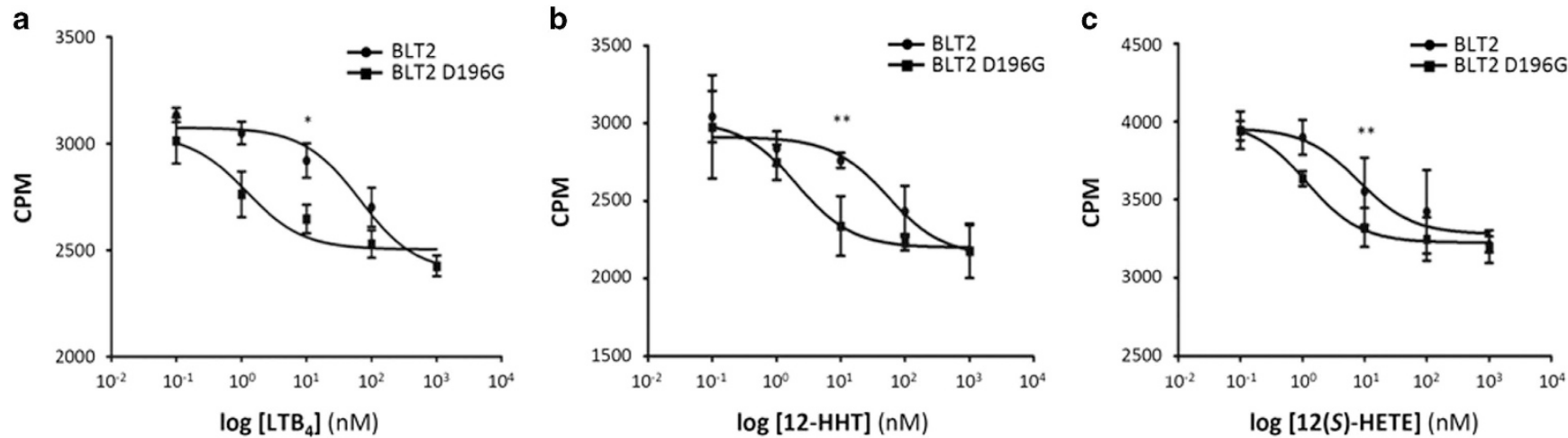

Figure 6 BLT2 D196G leads to enhanced ligand binding affinity. (a-c) Binding affinities of each ligand for BLT2 were measured as described in the Materials and Methods section. Binding parameters were determined with Prism software, and data are presented as means \pm s.d.; $n=3$ independent experiments. ${ }^{*} P<0.01$ and ${ }^{* *} P<0.005$ according to Student's $t$-test. The $t$-test was performed to determine whether the difference was significant between BLT2 and BLT2 D196G with a ligand concentration of $10 \mathrm{~nm}$.

our data indicated that BLT2 D196G increased BLT2-mediated motility after stimulation with various ligands, especially under low-dose conditions (Figures 2 and 3). Recent reports have suggested that BLT2 plays an important role in numerous inflammatory diseases, such as asthma and cancer. ${ }^{12,13,22-24}$ Thus, we hypothesized that populations with the BLT2 D196G SNP may be hypersensitive to variable diseases in which BLT2 signaling is involved. Future studies are clearly necessary to identify the function of BLT2 SNPs in human diseases.

Pharmacogenetics studies have investigated the correlation between genetic differences of GPCRs and their responses to ligands. ${ }^{33-38}$ In many cases, Gly substitution has been identified in GPCR variants, and its effect on cellular signaling and its role in physiology and disease have been described. For example, the $\beta 1$-adrenergic receptor SNP Arg389Gly decreases hyperfunctionality, and patients with the R389G variant exhibit decreased efficacy of $\beta$-blockers. ${ }^{39}$ In addition, the dopamine D3 receptor SNP Ser9Gly exhibits 4-fold enhanced ligandbinding affinity, ${ }^{7}$ inducing increased responses to the dopaminergic pathway. ${ }^{40}$ Similarly, in this study, we found that BLT2 D196G was more sensitive to its ligand under low-dose conditions. Furthermore, we observed that BLT2 D196G 
a

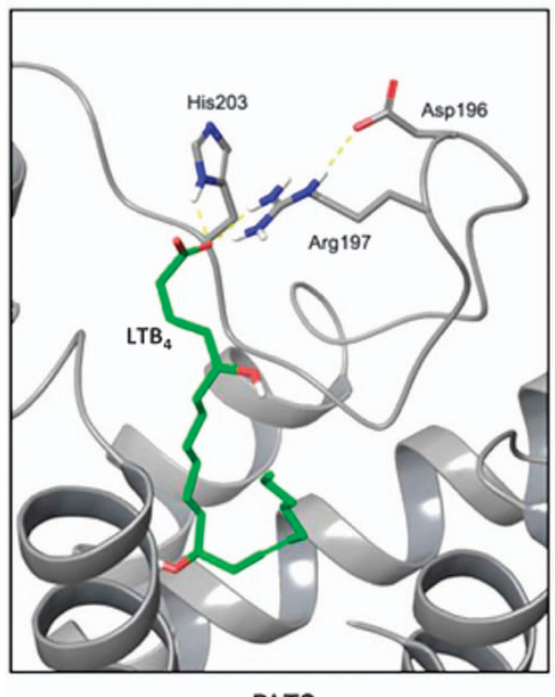

BLT2

(Asp-196 variant of BLT2) b

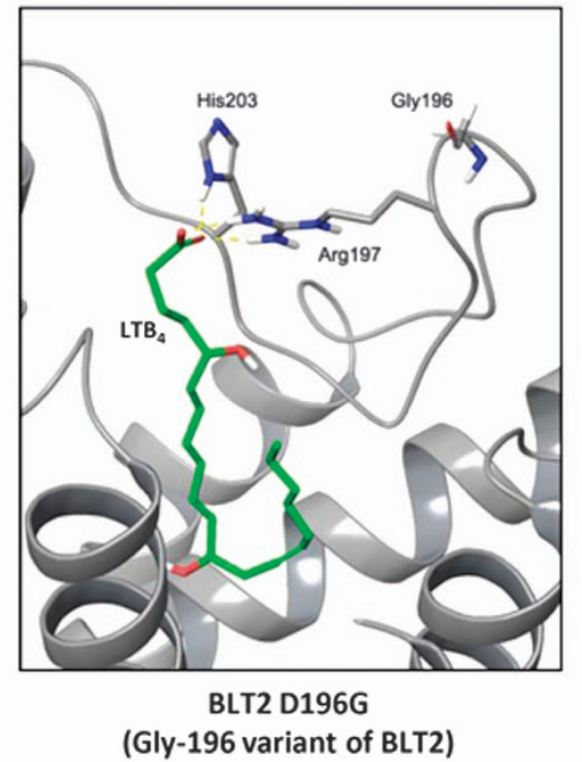

C

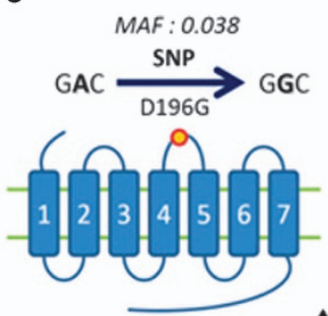

Ligand binding affinity $\uparrow$

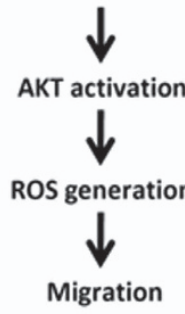

Figure 7 Predicted structures of BLT2 and BLT2 D196G in complex with LTB 4 . (a) BLT2 model. (b) BLT2 D196G model (gray ribbon: BLT2, green: $\mathrm{LTB}_{4}$, yellow dotted line: ionic interaction). (c) A scheme for the increasing effects of BLT2 D196G SNP on BLT2-mediated cell motility.

increased BLT2-mediated ROS generation and AKT activation under low-dose ligand stimulation (Figures 4 and 5). Therefore, we believe that BLT2 D196G is a Gly substitution observed in various GPCRs, and this SNP exhibits enhanced binding affinity to its ligands under low-dose stimulation.

The extracellular loop (ECLs) of GPCR can bind directly to docked ligands. Thus, ECLs of GPCRs permit or facilitate ligand entry into the transmembrane bundle, hold ligands within the transmembrane domain, direct ligands toward their binding site, change the shape of the binding pocket or directly bind orthosteric or allosteric ligands. ${ }^{41}$ Of the three ECLs, ECL2 is the largest and most structurally diverse. ECL2 is crucial for ligand recognition, control of ligand entry into the binding pocket, receptor subtype selectivity and receptorbinding kinetics. ${ }^{42}$ Indeed, 196 Asp is within the ECL2 of BLT2 and, in our ligand binding affinity study, we found that BLT2 D196G showed high ligand-binding affinity (Figure 6). We further predicted the detailed mechanism by which BLT2 D196G increases ligand affinity using a homology modeling assay. As shown in Figure 7, D196 was predicted to form ionic interactions with R197 to stabilize R197. However, in BLT2 D196G, R197 would undergo a conformational change because of the absence of the interaction with residue 196 and forms a closer and stronger ionic interaction with $\mathrm{LTB}_{4}$ (Figure 7). Consistent with this prediction, our modeling analysis demonstrated that $\mathrm{LTB}_{4}$ exhibits more favorable binding free energy in BLT2 D196G than BLT2, and the difference in predicted binding free energy $(\Delta \Delta \mathrm{G})$ was $-3.61 \mathrm{kcal} \mathrm{mol}^{-1}$. We hypothesize that this small and subtle free energy change is probably sufficient to trigger enhanced binding affinity under low-dose ligand conditions but is not sufficient to cause hyperaffinity when the doses of the ligands are above certain levels. Clearly, further analysis is necessary to understand fully the detailed mechanism of this SNP in terms of its effects on ligand binding or activation. On the basis of these results, we propose a model that describes the mechanism of the BLT2 D196G SNP (Figure 7c). In this model, BLT2 D196G SNP possesses enhanced ligand binding affinity, thus leading to hypersensitivity to its ligand (Figure 7c). Similar to our observations, recent studies have reported that ECL2 of GPCR plays a critical role in receptor activation and ligand efficacy. ${ }^{43}$ In addition, a recent report has shown that the aspartic acid in ECL2 of $\alpha_{1 B}$ adrenoceptor is important in the regulation of agonist binding. ${ }^{4}$

In conclusion, to the best of our knowledge, this report is the first to define the role of the BLT2 D196G SNP (rs1950504). We demonstrated that the BLT2 D196G SNP is hypersensitive to its ligand, thereby leading to enhanced BLT2-mediated cell motility under low-dose ligand conditions. Although further studies are needed to investigate the exact role of the BLT2 SNP in human physiopathology, we believe that this variant is likely to present a genetic basis for inter-individual differences in disease susceptibility and may inform the development of effective therapies for targeting BLT2.

\section{CONFLICT OF INTEREST}

The authors declare no conflict of interest.

\section{ACKNOWLEDGEMENTS}

This work was supported by Bio and Medical Technology Development Program grants (2012M3A9C1053532 and 2017M3A9D8063317) and a Mid-Career Researcher Program grant (2017R1A2B4002203) through the National Research Foundation 
(NRF) funded by the Ministry of Science, Information and Communication Technologies (ICT) and Future Planning of the Republic of Korea. This work was also supported by Basic Science Research (2015R1D1A1A01057757) through the NRF funded by the Ministry of Education, BK21 Plus Program (College of Life Sciences and Biotechnology, Korea University) and by a Korea University Grant.

\section{PUBLISHER'S NOTE}

Springer Nature remains neutral with regard to jurisdictional claims in published maps and institutional affiliations.

1 Cotton M, Claing A. G protein-coupled receptors stimulation and the contro of cell migration. Cell Signal 2009; 21: 1045-1053.

2 Reimann F, Gribble FM. G protein-coupled receptors as new therapeutic targets for type 2 diabetes. Diabetologia 2016; 59: 229-233.

3 Tang XL, Wang Y, Li DL, Luo J, Liu MY. Orphan G protein-coupled receptors (GPCRs): biological functions and potential drug targets. Acta Pharmacol Sin 2012; 33: 363-371.

4 Rask-Andersen M, Almen MS, Schioth HB. Trends in the exploitation of novel drug targets. Nat Rev Drug Discov 2011; 10: 579-590.

5 Srinivasan S, Clements JA, Batra J. Single nucleotide polymorphisms in clinics: fantasy or reality for cancer? Crit Rev Clin Lab Sci 2016; 53: 29-39.

6 Despotovic M, Stoimenov TJ, Stankovic I, Pavlovic D, Sokolovic D, Cvetkovic $T$ et al. Gene polymorphisms of tumor necrosis factor alpha and antioxidant enzymes in bronchial asthma. Adv Clin Exp Med 2015; 24: 251-256.

7 Krishnamoorthy S, Rajan R, Banerjee M, Kumar H, Sarma G, Krishnan S et al. Dopamine D3 receptor Ser9Gly variant is associated with impulse control disorders in Parkinson's disease patients. Parkinsonism Relat Disord 2016; 30: 13-17.

8 Funk CD. Prostaglandins and leukotrienes: advances in eicosanoid biology. Science 2001; 294: 1871-1875.

9 Yokomizo T, Kato K, Terawaki K, Izumi T, Shimizu T. A second leukotriene $\mathrm{B}(4)$ receptor, $\mathrm{BLT2}$. A new therapeutic target in inflammation and immunological disorders. J Exp Med 2000; 192: 421-432.

10 Park GS, Kim JH. LPS up-regulates ICAM-1 expression in breast cancer cells by stimulating a MyD88-BLT2-ERK-linked cascade, which promotes adhesion to monocytes. Mol Cells 2015; 38: 821-828.

11 Lee AJ, Cho KJ, Kim JH. MyD88-BLT2-dependent cascade contributes to LPS-induced interleukin-6 production in mouse macrophage. Exp Mol Med 2015; 47: e156.

12 Hennig R, Osman T, Esposito I, Giese N, Rao SM, Ding XZ et al. BLT2 is expressed in PanINs, IPMNs, pancreatic cancer and stimulates tumour cell proliferation. Br J Cancer 2008; 99: 1064-1073.

13 Cho KJ, Seo JM, Shin Y, Yoo MH, Park CS, Lee SH et al. Blockade of airway inflammation and hyperresponsiveness by inhibition of BLT2, a lowaffinity leukotriene B4 receptor. Am J Respir Cell Mol Biol 2010; 42: 294-303.

14 lizuka Y, Yokomizo T, Terawaki K, Komine M, Tamaki K, Shimizu T. Characterization of a mouse second leukotriene B4 receptor, mBLT2: BLT2-dependent ERK activation and cell migration of primary mouse keratinocytes. J Biol Chem 2005; 280: 24816-24823.

15 Choi JA, Kim EY, Song H, Kim C, Kim JH. Reactive oxygen species are generated through a BLT2-linked cascade in Ras-transformed cells. Free Radic Biol Med 2008; 44: 624-634.

16 Kim JY, Lee WK, Yu YG, Kim JH. Blockade of LTB4-induced chemotaxis by bioactive molecules interfering with the BLT2-Galphai interaction. Biochem Pharmacol 2010; 79: 1506-1515.

17 Wei JD, Kim JY, Kim JH. BLT2 phosphorylation at Thr355 by Akt is necessary for BLT2-mediated chemotaxis. FEBS Lett 2011; 585: 3501-3506.

18 Eun JC, Moore EE, Banerjee A, Kelher MR, Khan SY, Elzi DJ et al. Leukotriene b4 and its metabolites prime the neutrophil oxidase and induce proinflammatory activation of human pulmonary microvascular endothelial cells. Shock 2011; 35: 240-244.

19 Mathis SP, Jala VR, Lee DM, Haribabu B. Nonredundant roles for leukotriene B4 receptors BLT1 and BLT2 in inflammatory arthritis. $J$ Immunol 2010; 185: 3049-3056.
20 Sanchez-Galan E, Gomez-Hernandez A, Vidal C, Martin-Ventura JL, Blanco-Colio LM, Munoz-Garcia B et al. Leukotriene B4 enhances the activity of nuclear factor-kappaB pathway through BLT1 and BLT2 receptors in atherosclerosis. Cardiovasc Res 2009; 81: 216-225.

21 Park MK, Park Y, Shim J, Lee HJ, Kim S, Lee CH. Novel involvement of leukotriene $\mathrm{B}(4)$ receptor 2 through ERK activation by PP2A downregulation in leukotriene $\mathrm{B}(4)$-induced keratin phosphorylation and reorganization of pancreatic cancer cells. Biochim Biophys Acta 2012; 1823: 2120-2129.

22 Kim EY, Seo JM, Kim C, Lee JE, Lee KM, Kim JH. BLT2 promotes the invasion and metastasis of aggressive bladder cancer cells through a reactive oxygen species-linked pathway. Free Radic Biol Med 2010; 49: 1072-1081.

23 Seo JM, Park S, Kim JH. Leukotriene B4 receptor-2 promotes invasiveness and metastasis of ovarian cancer cells through signal transducer and activator of transcription 3 (STAT3)-dependent upregulation of matrix metalloproteinase 2. J Biol Chem 2012; 287: 13840-13849.

$24 \mathrm{Kim} \mathrm{H}$, Choi JA, Park GS, Kim JH. BLT2 up-regulates interleukin-8 production and promotes the invasiveness of breast cancer cells. PLOS ONE 2012; 7: e49186.

25 Park GS, Kim JH. Myeloid differentiation primary response gene 88 leukotriene B4 receptor 2 cascade mediates lipopolysaccharidepotentiated invasiveness of breast cancer cells. Oncotarget 2015; 6: 5749-5759.

26 Wei JD, Kim JY, Kim AK, Jang SK, Kim JH. RanBPM protein acts as a negative regulator of BLT2 receptor to attenuate BLT2-mediated cell motility. J Biol Chem 2013; 288: 26753-26763.

$27 \mathrm{Kim} \mathrm{M}$, Cho AE. Incorporating QM and solvation into docking for applications to GPCR targets. Phys Chem Chem Phys 2016; 18 : 28281-28289.

28 Yates A, Akanni W, Amode MR, Barrell D, Billis K, Carvalho-Silva D et al. Ensembl 2016. Nucleic Acids Res 2016; 44(D1): D710-D716.

29 Okuno T, lizuka Y, Okazaki H, Yokomizo T, Taguchi R, Shimizu T. 12(S)Hydroxyheptadeca-5Z, 8E, 10E-trienoic acid is a natural ligand for leukotriene B4 receptor 2. J Exp Med 2008; 205: 759-766.

30 Zhang F, Steinberg SF. S49G and R389G polymorphisms of the beta(1)adrenergic receptor influence signaling via the CAMP-PKA and ERK pathways. Physiol Genomics 2013; 45: 1186-1192.

31 Ahles A, Rodewald F, Rochais F, Bunemann M, Engelhardt S. Interhelical interaction and receptor phosphorylation regulate the activation kinetics of different human betal-adrenoceptor variants. J Biol Chem 2015; 290: 1760-1769.

32 Hoff M, Balfanz S, Ehling P, Gensch T, Baumann A. A single amino acid residue controls $\mathrm{Ca}^{2+}$ signaling by an octopamine receptor from Drosophila melanogaster. FASEB J 2011; 25: 2484-2491.

33 Insel PA, Tang CM, Hahntow I, Michel MC. Impact of GPCRs in clinical medicine: monogenic diseases, genetic variants and drug targets. Biochim Biophys Acta 2007; 1768: 994-1005.

34 Katara P, Chauhan S, Arora R, Saini P. A unilateral rectus sternalis muscle: rare but normal anatomical variant of anterior chest wall musculature. J Clin Diagn Res 2013; 7: 2665-2667.

35 Shastry BS. Further support for the common variants in complement factor $\mathrm{H}(\mathrm{Y} 402 \mathrm{H})$ and LOC387715 (A69S) genes as major risk factors for the exudative age-related macular degeneration. Ophthalmologica 2006; 220: 291-295.

36 Choi UY, Kang JS, Hwang YS, Kim YJ. Oligoadenylate synthase-like (OASL) proteins: dual functions and associations with diseases. Exp Mol Med 2015; 47: e144.

37 Kitteringham NR, Palmer L, Owen A, Lian LY, Jenkins R, Dowdall S et al. Detection and biochemical characterisation of a novel polymorphism in the human GSTP1 gene. Biochim Biophys Acta 2007; 1770: 1240-1247.

38 Hu HJ, Jin EH, Yim SH, Yang SY, Jung SH, Shin SH et al. Common variants at the promoter region of the APOM confer a risk of rheumatoid arthritis. Exp Mol Med 2011; 43: 613-621.

39 McCrink KA, Lymperopoulos A. beta1-adrenoceptor Arg389Gly polymorphism and heart disease: marching toward clinical practice integration. Pharmacogenomics 2015; 16: 1035-1038.

40 Jeanneteau F, Funalot B, Jankovic J, Deng H, Lagarde JP, Lucotte G et al. A functional variant of the dopamine $D 3$ receptor is associated with risk and age-at-onset of essential tremor. Proc Natl Acad Sci USA 2006; 103 : 10753-10758. 
41 Wheatley M, Wootten D, Conner MT, Simms J, Kendrick R, Logan RT et al. Lifting the lid on GPCRs: the role of extracellular loops. $\mathrm{Br} J$ Pharmacol 2012; 165: 1688-1703.

42 Woolley MJ, Conner AC. Understanding the common themes and diverse roles of the second extracellular loop (ECL2) of the GPCR super-family. Mol Cell Endocrinol 2017; 449: 3-11.

43 Seibt BF, Schiedel AC, Thimm D, Hinz S, Sherbiny FF, Muller CE. The second extracellular loop of GPCRs determines subtypeselectivity and controls efficacy as evidenced by loop exchange study at A2 adenosine receptors. Biochem Pharmacol 2013; 85: 1317-1329.

44 Campbell AP, MacDougall IJ, Griffith R, Finch AM. An aspartate in the second extracellular loop of the alpha(1B) adrenoceptor regulates agonist binding. Eur J Pharmacol 2014; 733: 90-96. (c) (1) (2) (2)

This work is licensed under a Creative Commons Attribution-NonCommercial-ShareAlike 4.0 International License. The images or other third party material in this article are included in the article's Creative Commons license, unless indicated otherwise in the credit line; if the material is not included under the Creative Commons license, users will need to obtain permission from the license holder to reproduce the material. To view a copy of this license, visit http:// creativecommons.org/licenses/by-nc-sa/4.0/

(C) The Author(s) 2017 